\title{
PROCEDIMIENTO DE MODELADO ISA S88 PARA EJECUCIÓN DE ÓRDENES DE PRODUCCIÓN BASADAS EN RÉCIPES
}

\author{
ISA S88 MODELING PROCEDURE FOR EXECUTION OF PRODUCTION ORDER BASED ON \\ RECIPES
}

\author{
Werner Yamid Serna Quilindo \\ Ingeniero en Automática Industrial, Universidad del Cauca, FIET, Popayán, Colombia \\ wserna@unicauca.edu.co \\ Diana Cecilia Vergara González \\ Ingeniera en Automática Industrial, Universidad del Cauca, FIET, Popayán, Colombia \\ dcgonzales@unicauca.edu.co \\ Juan Fernando Flórez Marulanda \\ Docente, Universidad del Cauca, Departamento de Electrónica, Instrumentación y Control, \\ miembro Grupo I+D en Automática Industrial, Popayán, Colombia \\ jflorez@unicauca.edu.co
}

Fecha de recepción: 15 de septiembre de 2011

Fecha de aprobación: 19 de diciembre de 2011

\begin{abstract}
RESUMEN
Este documento propone y desarrolla un procedimiento organizado para realizar el modelado de la norma ISA S88, partes 1 y 2, en la ejecución de órdenes de producción basadas en récipes, aplicado a un caso de estudio. Dicho procedimiento proporciona pautas para editar los modelos físico y de control procedimental en las herramientas del software industrial, de manera que dicha edición se haga de una forma adecuada, precisa y organizada. Además, se incluyen pasos que permiten construir diagramas de tuberías e instrumentación (P\&ID), de tal forma que tanto los modelos de proceso, físico y de control procedimental, establecidos en ISA S88, así como el diagrama P\&ID sean diseñados con la misma información y sean coherentes entre sí.
\end{abstract}

Palabras clave: ISA S88, modelado, récipes, estructura de datos, lineamientos de lenguaje.

\begin{abstract}
This paper proposes and develops a systematic procedure for carrying out the modeling of the ISA S88 for execution of production orders based on recipes, applied to a case study. This procedure provides guidelines for the issue of physical models and procedural control in industrials software tools, so that this issue be conducted in a proper, accurate and organized. Also included the steps that allow the construction of piping and instrumentation diagrams (P\&ID) so that both the process models, physical and procedural control, established in ISA S88,
\end{abstract}


and the P\&ID are designed with the same information and so as to have consistency between each of them.

Keywords: ISA S88, modeling, recipes, data structure, guidelines for language.

\section{INTRODUCCIÓN}

La integración industrial ofrece grandes ventajas a la industria, sin embargo, el camino hacia la integración es y ha sido más complicado de lo que parece. Ya desde principios de la década del 90, instituciones serias y doctas en el ramo de la automatización, como la Sociedad Internacional de Automatización (ISA), observaron esta dificultad en la producción y trabajaron para crear un protocolo de comunicación que permitiera unir los sistemas de gestión con el nivel de planta, y entonces propusieron los estándares ANSI/ISA 88 y 95 [1],[2].

En términos sencillos, la norma S88 desarrolló una metodología batch que permite a la industria crear formas estándares para automatizar la producción batch y al mismo tiempo, reducir tanto la complejidad como los costos asociados a los sistemas propietarios. La S88 indica que se debe analizar por separado, las capacidades de los equipos de la planta (modelo físico), y los procedimientos requeridos para realizar el proceso (modelo procedimental) [3]. El estándar pretende también aprovechar al máximo, la capacidad instalada y otorgar flexibilidad al momento de editar los récipes ${ }^{1}$ por parte de las personas responsables de la formulación o de las herramientas software diseñadas para tal fin. De hecho, se puede tener los mismos récipes para varias plantas de producción, lo cual facilita la migración y validación de las mismas entre diferentes centros de producción [1].

ISA S88 ha sido la base principal para desarrollar herramientas software encargadas de automatizar procesos de producción basados en récipes [4], y se ha reportado variedad de trabajos, usando la norma [5],[6],[7] y [8]. Sin embargo, en el momento de realizar el modelado de dicha norma y su aplicación en herramientas software, se observa que en el estándar se describen pautas y definiciones para cada uno de los componentes que hacen parte de los modelos, pero así mismo, dicho estándar no es claro con respecto de lo que abarca dentro del proceso cada una de estas definiciones, generando diversas problemáticas que no han sido reportadas en la literatura ni mucho menos atendidas. Por lo anterior, en el presente trabajo se aporta un procedimiento que proporciona una base para aplicar la ISA S88, procedimiento con el cual no se cuenta siquiera en las empresas integradoras [9], para definir los componentes de los tres modelos $\mathbf{8 8}$ de una manera normalizada, y de forma que la información consignada en ellos, esté relacionada. La ausencia de este procedimiento ha conllevado a que los modelos sean definidos de manera independiente y como consecuencia, se presentan incoherencias en ellos.

\footnotetext{
${ }^{1}$ Récipe: Conjunto de información necesaria que en forma única define los requerimientos de fabricación para un producto específico. Existen cuatro tipos de récipes definidos en la norma: general, de sitio, maestro y de control [1].
} 
En el presente trabajo, se identificaron dos problemáticas que se encuentran relacionadas con la ejecución de récipes a partir de sistemas software que son atendidas por el procedimiento propuesto: la primera hace referencia a la definición de las propiedades de los materiales y los equipos, información necesaria para la ejecución y simulación del proceso Batch; esta información se define actualmente, según el modelo de materiales y el modelo de equipos de la norma ISA S95 [10]. Sin embargo, el enfoque de dicho estándar es el nivel de gestión que implica que la información modelada sea menos específica y orientada a otro tipo de actividades diferentes al proceso productivo.

La segunda problemática identificada, radica en el uso de componentes software para edición de récipes que contienen algunos de los paquetes Batch comerciales, como los desarrollados por Rockwell Automation, Wonderware y Siemens, entre otros. Dicho componente software permite la edición del procedimiento de récipe maestro a partir del modelo PFC (por sus siglas en inglés, Procedural Function Chart) [1 1],[12] definido en la norma ISA S88.02. En la actualidad es práctica común que no se realice la edición del procedimiento de récipe maestro como tal ni el diagrama PFC, lo que se edita en la herramienta es el modelo de control procedimental, que es sólo un componente del récipe maestro. Estas herramientas software no tienen en cuenta los récipes en la definición del batch, esto conlleva a suponer información que debería estar contenida en dicho récipe, y ocasionar incoherencias entre la información obtenida del proceso y la información definida en las herramientas.

El objetivo principal del presente trabajo es proponer un procedimiento sistemático soportado en ISA S88, para implementar un proceso tipo batch en herramientas software comerciales. Inicialmente, estudia la definición del procedimiento que permite realizar los modelos de proceso, físico y de control procedimental y el diagrama de tubería e instrumentación (P\&ID), de un proceso caso de estudio. Después se establecen especificaciones de materiales y equipos, y se definen las bases para construir el diagrama PFC que representa los elementos procedimentales, con base en la estructura de datos y en los lineamientos de lenguaje definidos en la parte 2 de ISA S88. Para finalizar, se realiza la aplicación del procedimiento, las especificaciones de materiales y la construcción del diagrama PFC a un segmento del proceso caso de estudio: Adecuación de Leche, en donde se hace un precalentamiento y preparación de la leche pasteurizada, con el fin de establecer condiciones iniciales y al final, se consignan el análisis y los resultados.

\section{PROCEDIMIENTO DE MODELADO ISA S88}

Para definir los modelos de proceso, físico y de control procedimental, se debe tener en cuenta las definiciones establecidas por ANSI en su estándar ISA S88.01 [1]:

1. Etapa de proceso: parte de un proceso que opera independiente de otras etapas de proceso. Es el resultado de una secuencia planeada de cambios físicos o químicos en el material que está siendo procesado. 
2. Operaciones de Proceso: Conforman las etapas de proceso y representan actividades especializadas de procesamiento que resultan en un cambio físico o químico del material que está siendo procesado; pueden establecerse en un orden específico que se desarrolla para completar una etapa.

3. Acciones de Proceso: Son las subdivisiones de las operaciones de proceso que desarrollan un conjunto de actividades menores para llevar a cabo el procesamiento requerido por cada operación de proceso.

4. Unidad: Conjunto de equipos de procesamiento y control necesarios para desarrollar actividades mayores de proceso. Operan relativamente independientes unas de otras.

5. Módulos de equipo: Una o varias piezas de equipo que pueden llevar a cabo un número finito de tareas específicas. Físicamente, pueden estar formados por módulos de control y otros módulos de equipo; deben ser parte de una unidad.

6. Módulos de control: Son los equipos que llevan a cabo las acciones de control básico. Típicamente, son una colección de sensores, válvulas, motores, actuadores y otros módulos de control que permiten establecer y mantener un estado específico de los equipos y procesos.

7. Procedimiento: Es el nivel superior en la jerarquía y define la estrategia para llevar a cabo una acción de procesamiento importante como hacer un batch. Está definido en términos de un conjunto ordenado de procedimientos de unidad.

8. Procedimiento de Unidad: Consiste en un conjunto ordenado de operaciones que causa una secuencia de producción contigua que tendrá lugar dentro de una unidad.

9. Operación: Conjunto ordenado de fases que define una secuencia de procesamiento más importante que lleva el material en proceso de un estado a otro, y por lo general incluye un cambio físico o químico.

10. Fase: Es el elemento de control procedimental que puede llevar a cabo una tarea orientada a proceso. Una fase puede subdividirse en partes más pequeñas. La intención de la fase es ocasionar o definir una acción orientada a proceso.

11. Récipe maestro: Es un nivel de récipe dirigido a la célula de proceso o un subconjunto de equipos de la célula de proceso. Un récipe maestro puede ser derivado desde un récipe general o un récipe de sitio.

12. Récipe de control: Es una copia de una versión específica de un récipe maestro que se modifica en caso necesario, con la programación y la información operativa específica para un único batch. Contiene información necesaria del proceso de un producto específico para fabricar un batch en particular.

A continuación se desarrolla el procedimiento propuesto por los autores en [13], para mejorar la aplicación de la norma ISA S88. Inicialmente, se evidencia el procedimiento para definir modelos de la parte 1 del mismo estándar: modelo de proceso, físico y de control procedimental, además del récipe maestro. Después se muestra el procedimiento que se llevó a cabo para realizar las especificaciones de materiales y equipos, y el diagrama para editar el procedimiento de récipe en un componente software desarrollado para tal fin. 


\subsection{PROCEDIMIENTO DE APLICACIÓN NORMA $\$ 88.01$}

En esta sección se diseña el procedimiento que provee una guía para definir los modelos ISA S88.01, para solucionar las dificultades ya identificadas en la definición de dichos modelos. El procedimiento propuesto permite reorganizar la información del proceso mediante un acondicionamiento del mismo, y a partir de él, se diseñan los modelos y los diagramas necesarios, de tal forma que la información se refleje en los modelos, y que estos sean consecuentes entre sí.

Acondicionamiento del proceso para aplicación de S88. Inicialmente, se realiza un análisis del proceso que se pretende modelar. Después de conocer en detalle el proceso, se puede iniciar el diseño del modelo batch. Para tal fin, se debe tener en cuenta ciertos pasos que permiten desarrollar un procedimiento de diseño adecuado, de tal forma que se cumplan los requerimientos establecidos en el estándar S88.01 de ISA y se logre definir modelos relacionados que faciliten la ejecución de procesos batch, con herramientas software, además del diagrama P\&ID que permita conocer las relaciones funcionales entre la instrumentación, los equipos y las tuberías por donde circulan los materiales presentes en la planta.

Los pasos del acondicionamiento del proceso, para aplicar ISA S88 los proponen los autores a partir del análisis de [14] y [15], y se listan a continuación:

a. Realizar el diagrama de flujo de proceso donde se pueda visualizar el flujo de la materia prima y el producto, los materiales aditivos y demás que estén presentes en el mismo.

b. Basarse en los conceptos y terminología de la norma ISA S88.01 para la definición de los componentes del modelo de proceso, modelo físico y modelo de control procedimental.

c. Para definir el P\&ID, se sugiere generar primero un listado de etapas de proceso; después, definir las unidades según la norma, de tal forma que se tenga una unidad asociada a cada una de las etapas de proceso.

d. Una vez definidas las etapas y unidades, se procede a describir cada una de las etapas: deberá contener material entrante, material saliente, aditivos utilizados $y$, el proceso llevado a cabo sobre el material.

e. Describir cada una de las unidades: deberá contener la función de los equipos involucrados en la unidad.

f. Determinar los módulos de equipo y sus correspondientes módulos de control; para determinar los módulos de control se debe hacer un análisis adicional donde se definen variables controladas manipuladas; posibles disturbios presentes en el proceso desarrollado en cada módulo de equipo, clasificación del disturbio en crítico, no crítico o no existente, y plantear un esquema de control para manejar dichos disturbios.

g. Definir las operaciones de proceso que se llevan a cabo dentro de cada etapa, y que deben estar asociadas a los módulos de equipo; y, las acciones de proceso dentro de cada operación respectiva, teniendo en cuenta que son acciones menores que en conjunto, permitirán realizar la operación.

h. A partir de las descripciones realizadas en cada etapa de proceso y unidades, se procede a realizar el modelo de proceso y el modelo físico. 
i. Una vez realizados los dos modelos mencionados y a partir de las definiciones de la norma ISA S88, se debe determinar los componentes para el modelo de control procedimental y realizarlo.

j. Basándose en el diagrama de flujo de proceso o PFD (por sus siglas en inglés Process Flow Diagram), los módulos de control y los esquemas de control sugeridos, se debe construir el P\&ID de la planta, de forma que la información reflejada en los modelos del proceso, coincida con la representada en el diagrama.

k. Finalmente, con base en la información del proceso y el modelo de control procedimental, se definen los récipes maestro y de control.

Al seguir este procedimiento, se obtendrán los modelos definidos por la norma ISA S88.01 y el P\&ID con un mismo análisis de la información del proceso. Sin embargo, se puede presentar que el análisis de la información se haga a un proceso con cierto nivel de automatización y ya cuente con diagramas de tuberías e instrumentación; en este caso, no es necesario realizar los pasos $f$ y $j$, pero se recomienda realizar una revisión del diagrama existente, con el fin de chequear inconsistencias entre dicho diagrama y los modelos, para corregirlas en el caso dado.

\subsection{PROCEDIMIENTO PARA APLICAR LA NORMA ISA S88.02 EN EL USO DE HERRAMIENTAS DE EJECUCION BATCH}

Esta sección se desarrolla con el fin de proponer soluciones a las problemáticas ya descriptas, en cuanto al uso de herramientas software diseñadas para editar los modelos de la ISA S88.01.

Para la primera problemática, se propone redefinir las especificaciones de materiales y equipos, basándose no en ISA 95, sino en la estructura de datos contenida en la parte 2 de la ISA S88, ya que esta permite definir dichos requerimientos a nivel de planta. Sin embargo, para una integración hacia los niveles empresariales, se debe utilizar los modelos de ISA 95, teniendo en cuenta las definiciones de las especificaciones de materiales y equipos, realizadas bajo los criterios de ISA 88. Dicha solución se analiza en las secciones 0 y 1.4, que corresponden a la estructura de datos propuesta por ISA S88 y a la aplicada, respectivamente.

Para la segunda problemática, se propone realizar el diagrama PFC del proceso caso de estudio, basado en el procedimiento de récipe, siguiendo los lineamientos de lenguaje establecidos en la ISA S88 parte 2, lo cual se estudia en las secciones 0 y 1.5, que corresponden a las pautas establecidas por ISA y a su aplicación.

Estructura de datos definida en ISA S88.02 [16]. Define modelos de datos que especifican un conjunto de objetos, atributos y sus relaciones básicas, y cubren los conceptos de la ISA S88.01 en un mayor nivel de abstracción. El propósito de usar estos modelos, es proveer un punto inicial para desarrollar especificaciones para herramientas software que se encargan de la aplicación de ISA S88.01. 
Se definen las estructuras de datos que permiten complementar la información necesaria para la simulación del Batch, teniendo en cuenta los componentes del récipe maestro, como el encabezado, la fórmula, y los requerimientos de equipos. Esta definición se realiza, siguiendo los pasos a hasta c, que se listan a continuación:

a) El encabezado está contenido en los atributos de la propia entidad de récipe; y se define un modelo de datos para el récipe orientado a la célula de proceso con la información del encabezado, tal como se muestra en la Tabla 1.

Tabla 1. Estructura de Datos para Definición de Entidad de Récipe

\begin{tabular}{|c|c|}
\hline Nombre & Entidad de récipe \\
\hline Descripción Funcional & Breve descripción de acuerdo con la función dentro del proceso. \\
\hline \multicolumn{2}{|r|}{ Atributos } \\
\hline ID Entidad Récipe & Provee identificación única para el récipe. \\
\hline ID Célula de Proceso & Identifica el equipo para el cual fue definido el récipe. \\
\hline Récipe de Producción & Identifica el proceso al cual está orientado. \\
\hline Versión de récipe & Identifica la versión del récipe. \\
\hline Autor & Identifica la persona que creó esta versión del récipe. \\
\hline
\end{tabular}

b) Después se define el modelo de datos del récipe maestro para el proceso de producción, que debe contener toda la información registrada en el modelo de récipe maestro, tal como se muestra en la Tabla 2.

Tabla 2. Estructura de datos para definición de récipe maestro

\begin{tabular}{|l|l|}
\hline \multicolumn{1}{|c|}{ Nombre } & \multicolumn{1}{c|}{ Récipe } \\
\hline Descripción Funcional & \multicolumn{1}{|c|}{ Areve descripción de acuerdo la función dentro del proceso. } \\
\hline \multicolumn{2}{|l|}{} \\
\hline ID Récipe & Identifica el récipe. \\
\hline Versión Récipe & Identifica la versión del récipe. \\
\hline Fecha Versión & Identifica la fecha de creación o modificación de la versión del récipe. \\
\hline Fecha Aprobado & Identifica la fecha cuando esa versión del récipe fue aprobada. \\
\hline Fecha Efectiva & Identifica la fecha cuando esa versión del récipe fue usada. \\
\hline Fecha de expiración & Identifica la fecha cuando el récipe expira. \\
\hline ID Producto & $\begin{array}{l}\text { Identifica el producto o familia de productos que sería creado en la ejecución de esta } \\
\text { versión del récipe (ejemplo: cerveza Premium). }\end{array}$ \\
\hline Autor & Identifica la persona que creó esta versión del récipe. \\
\hline Aprobado por & Identifica la persona o sistema que aprobó esta versión del récipe. \\
\hline Descripción & $\begin{array}{l}\text { Describe esta versión del récipe y/o producto (mejor cerveza Premium de Carolina del } \\
\text { Norte.) }\end{array}$ \\
\hline Estado & $\begin{array}{l}\text { Define el estado de la información (ejemplo: aprobado para producción, aprobado } \\
\text { para prueba, no aprobado, inactivo, obsoleto). }\end{array}$ \\
\hline Fuente: Adaptado de [16], mayo de 2011
\end{tabular}


c) La categoría fórmula de la parte 1 de la norma ISA S88, está modelada como un conjunto de parámetros de objetos. ISA 588.02 se refiere a los materiales como parámetros de récipe, y se dividen en tres tipos de parámetros: entradas de proceso, parámetros de proceso, salidas de proceso. Los tres tipos de parámetros poseen la misma estructura de datos que se define como se muestra en la Tabla 3.

Tabla 3. Estructura de datos para definición de parámetros

\begin{tabular}{|l|l|}
\hline \multicolumn{1}{|c|}{ Nombre } & \multicolumn{1}{c|}{ Parámetros } \\
\hline Descripción Funcional & Breve descripción de acuerdo con la función dentro del proceso. \\
\hline \multicolumn{2}{|c|}{ Atributos } \\
\hline ID Parámetro & \multicolumn{1}{|c|}{ Provee identificación única. } \\
\hline Tipo de Parámetro & $\begin{array}{l}\text { Especifica cómo se interpretan los valores de los parámetros (ejemplo: } \\
\text { constante, ecuación de referencia). }\end{array}$ \\
\hline Descripción & Describe los parámetros o uso de los parámetros. \\
\hline Unid. de Ingeniería & Identifica las unidades de medida de ingeniería para el valor. \\
\hline Valor & $\begin{array}{l}\text { Contiene el valor del parámetro. Si el valor es una relación, contiene la } \\
\text { ecuación o cualquier relación que tengan los parámetros entre sí. }\end{array}$ \\
\hline Escala & $\begin{array}{l}\text { Específica la regla de escalizado. Caso más simple: escalizado o no } \\
\text { escalizado con tamaño de referencia del batch. }\end{array}$ \\
\hline Uso & $\begin{array}{l}\text { Especifica el parámetro como una entrada de proceso, salida de } \\
\text { proceso o parámetro de proceso. }\end{array}$ \\
\hline Fuente: Tomado de [16], mayo de 2011 \\
\hline
\end{tabular}

Para definir los requerimientos de cada equipo, se debe realizar inicialmente una instancia del equipo, como se ve en la Tabla 4 y después, una instancia de la(s) propiedad(es) del mismo como en la Tabla 5.

Tabla 4. Estructura de datos para entidad de equipo

\begin{tabular}{|l|l|}
\hline \multicolumn{1}{|c|}{ Nombre } & \multicolumn{1}{c|}{ Entidad de equipo } \\
\hline Descripción funcional & $\begin{array}{l}\text { Una colección de procesamiento físico y equipo de control y control de } \\
\text { equipo que se agrupan para desempeñar una cierta función de control } \\
\text { o un conjunto de funciones de control. }\end{array}$ \\
\hline \multicolumn{2}{|c|}{ Atributos } \\
\hline ID Entidad equipo & \multicolumn{1}{|c|}{ Provee identificación única } \\
\hline Nivel de equipo & $\begin{array}{l}\text { Especifica el nivel de jerarquía físico (ejemplo: célula de proceso, } \\
\text { unidad, módulo equipo, módulo de control). }\end{array}$ \\
\hline Modo & Indica el modo actual de la entidad de equipo. \\
\hline Estado & Indica el estado actual de la entidad de equipo. \\
\hline Fuente: Tomado de [16], mayo de 2011 \\
\hline
\end{tabular}


Con el procedimiento de definición para la estructura de datos, se puede establecer las especificaciones de materiales y equipos para cualquier proceso tipo batch.

Lineamientos de lenguaje definidos en ISA S88.02. ISA S88.02 describe las pautas para crear los diagramas PFC, a partir de símbolos y reglas que se necesitan para un proceso batch determinado. ISA $\mathbf{5 8 8 . 0 2}$ define los símbolos gráficos relacionados con el procedimiento de récipe y las reglas para su uso [16].

Símbolos de diagrama PFC [16]: El diagrama PFC está definido como un conjunto de símbolos para: Elementos, Puntos de inicio y final, Transiciones de récipe y, Estructuras básicas como conexiones directas, selecciones de secuencia y secuencias simultáneas. A continuación, se describe cada símbolo definido anteriormente:

Tabla 5. Estructura de datos para propiedades de equipo

\begin{tabular}{|l|l|}
\hline \multicolumn{1}{|c|}{ Nombre } & \multicolumn{1}{c|}{ Propiedad de equipo } \\
\hline Descripción funcional & Breve descripción de acuerdo con la función dentro del proceso. \\
\hline \multicolumn{2}{|c|}{ Atributos } \\
\hline ID Propiedad & Provee identificación única \\
\hline Valor & Identifica el valor nominal de la propiedad. \\
\hline Rango & Define límites o restricciones que están relacionadas con el valor. \\
\hline Unid. de ingeniería & Define las unidades de ingeniería de la propiedad. \\
\hline Descripción & Describe el tipo de propiedad del equipo. \\
\hline Fuente: Tomado de [16], mayo de 2011
\end{tabular}

Símbolos de Elementos. Se utilizan para representar una fase de récipe, una operación de récipe, un procedimiento de unidad de récipe o un procedimiento de récipe. La identificación de elementos de procedimiento se muestra en la Figura 1.

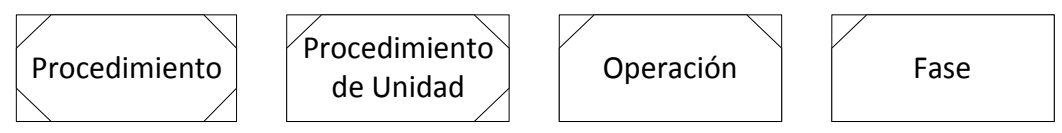

Figura 1. Elementos de procedimiento diagrama PFC

Fuente: Tomado de [16], mayo de 2011

Un elemento de procedimiento por encima del nivel de una fase, puede representar una encapsulación de otros elementos de procedimiento en el siguiente nivel inferior en la jerarquía de control de procedimiento. Un elemento de procedimiento que representa un encapsulado, donde el elemento de procedimiento de récipe de nivel más bajo no se muestra, se identifica con un signo más (+) en la esquina superior derecha del rectángulo, representando el elemento procedimental (Figura 2). Los símbolos de elemento procedimental que exponen la encapsulación, se identifican por un signo menos (-). 


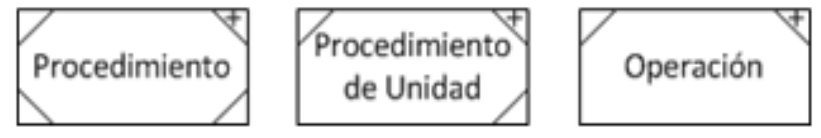

Figura 2. Elementos procedimentales que encapsulan elementos de nivel más bajo

Fuente: Tomado de [16], mayo de 2011

Símbolos para puntos de inicio y final. Los diagramas PFC deben tener por lo menos un símbolo de inicio para designar el comienzo de cada PFC, y por lo menos, un símbolo final para designar el final de cada diagrama PFC (Figura 3).
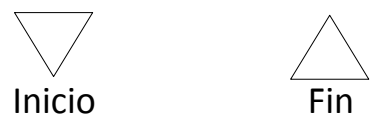

Figura 3. Símbolos inicio y fin de los diagramas PFC

Fuente: Tomado de [16], mayo de 2011

Símbolos para transiciones de récipe. Los diagramas PFC usan dos tipos de transiciones: las implícitas y las explícitas.

Transiciones implícitas: En este caso, la conexión entre las entidades de récipe es directa y se representa con una única línea entre las entidades de récipe. Esta conexión indica una transición cuya única condición es que las entidades directamente anteriores a la transición, hayan terminado su ejecución (Figura 4a).

a)

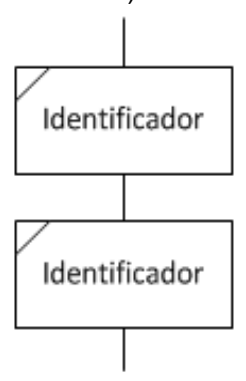

b)

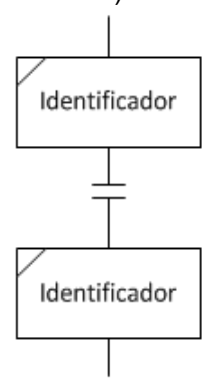

Figura 4. Transiciones de récipe: a) Transición implícita y b) Transición explícita

Fuente: Tomado de [16], mayo de 2011

Transiciones explícitas: Ocurren cuando la conexión entre las entidades de récipe es directa y se representa con una única línea entre las entidades de récipe, la cual tiene dos barras cortas perpendiculares a la línea de enlace, (Figura 4 b). Esta transición está definida por una expresión que se evalúa como verdadera o falsa. 
Símbolos para estructuras básicas. Las estructuras definen el camino previsto de ejecución de los elementos de récipe. El caso más simple es una serie de elementos de procedimiento de récipe que se activarán uno tras otro. Pero existen dos estructuras más complejas.

a)

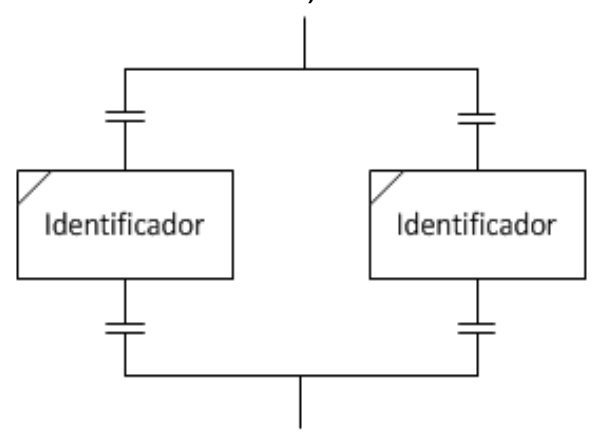

b)

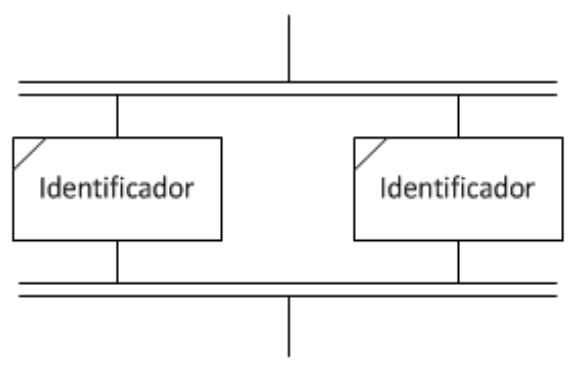

Figura 5. Símbolos de estructuras básicas: a) Selección de secuencia y b) Secuencia simultánea

Fuente: Tomado de [16], mayo de 2011

Selección de secuencia: Cada rama de una selección de secuencia comenzará con una transición explícita. Sólo debe seleccionarse una secuencia del conjunto de secuencias. Las transiciones se evalúan en prioridad de izquierda a derecha (Figura 5 a).

Secuencia simultánea: Hay un camino de ejecución para cada vía debajo del inicio de la selección. Todos los caminos de ejecución deben ser accedidos. La transición que sigue inmediatamente a las líneas paralelas, se evalúa sólo cuando todas las entidades que preceden inmediatamente a las líneas paralelas se hayan completado (Figura 5 b).

Diagrama PFC del procedimiento de récipe. Una vez analizados los componentes gráficos del diagrama PFC, se definen los lineamientos de lenguaje, basándose en el parágrafo 6 de la norma ISA S88.02, para construir del diagrama PFC del procedimiento del récipe maestro en estudio, según los cuatro pasos listados a continuación:

a) Para realizar el diagrama PFC de una manera sencilla, se inicia con las fases descritas en el procedimiento de récipe maestro.

b) Una vez se tengan las fases, se prosigue con las operaciones, de manera que se determine qué fases se encuentran encapsuladas en cada operación.

c) Diseñado el diagrama PFC con las operaciones, se construyen los procedimientos de unidad, de manera que encapsulen sus respectivas operaciones según la definición del procedimiento de récipe maestro.

d) Finalmente, se construye el diagrama PFC final con el procedimiento, que deberá contener los procedimientos de unidad definidos. 
Cada uno de los componentes del diagrama: fases, operaciones, procedimientos de unidad y procedimiento, se encuentran definidos en el Récipe Maestro. Con estos pasos, se construye el diagrama PFC para cualquier proceso batch.

\section{APLICACIÓN A UN CASO DE ESTUDIO}

En esta sección, se realiza el proceso de aplicación de los procedimientos establecidos en las secciones 1.1 y 1.2, para el segmento Adecuación de Leche del proceso de fabricación de yogurt.

\subsection{MODELADO DE PROCESO CASO DE ESTUDIO}

En la presente sección, se realiza el procedimiento propuesto para la aplicación de ISA S88.01.

Diagrama de flujo de proceso: El diagrama de flujo de proceso, (Figura 6), es un esquema que permite conocer las características físicas del segmento Adecuación de Leche. En él, se debe ver el camino de transformaciones físicas y químicas a las cuales se ve sometida la leche pasteurizada en su proceso de adecuación [17].

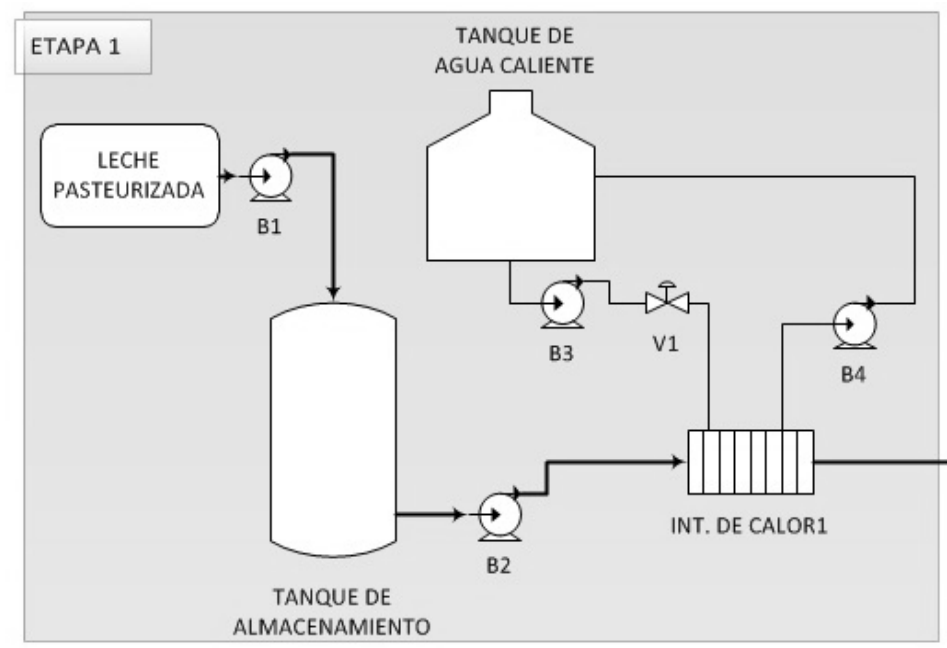

Figura 6. Diagrama de flujo de segmento de proceso Adecuación de Leche

Inicialmente, se realiza una adecuación de la leche (Etapa 1): se recibe la leche pasteurizada que, por medio de la bomba B1, se transfiere al tanque de almacenamiento; luego se transfiere por medio de la bomba B2 al intercambiador de calor 1, donde se calienta a $42-43{ }^{\circ} \mathrm{C}$ con ayuda del agua caliente que se encuentra almacenada en el Tanque de agua caliente; esto se hace por medio de la válvula V1 y la bomba B3 que suministra una determinada cantidad de agua al intercambiador de calor 1 . El rango de temperatura ayuda a la propagación del cultivo 
bacteriológico en la leche. Una vez el agua caliente pase por el intercambiador de calor 1, se retorna al tanque de agua caliente por medio de la bomba B4.

Definición de etapas y unidades: Según las definiciones de etapa y unidad, establecidas en la norma ISA S88, y el punto c del procedimiento de la sección 1.1, se puede determinar las etapas y unidades del proceso de fabricación de yogurt, de acuerdo con el siguiente listado:

\section{Etapas}

Etapa 1: Adecuación de leche.

Etapa 2: Fermentación.

Etapa 3: Enfriamiento de fermentado.

Etapa 4: Homogeneización.

Etapa 5: Enfriamiento de yogurt.

Etapa 6: Envasado.

\section{Unidades}

Unidad 1: Unidad de adecuación.

Unidad 2: Unidad de fermentación.

Unidad 3: Unidad de enfriamiento de fermentado.

Unidad 4: Unidad de homogeneización.

Unidad 5: Unidad de enfriamiento de yogurt.

Unidad 6: Unidad de envasado.

\section{Descripción de Etapas}

Etapa 1: Adecuación de leche [17]: Al recibir la leche pasteurizada, se deposita en el tanque de almacenamiento. En esta parte del proceso, se debe especificar la cantidad de leche que se proveerá al proceso subsiguiente, según la cantidad de yogurt que se desee producir.

La leche proveniente del tanque de almacenamiento debe ser sometida a un calentamiento de $42-43^{\circ} \mathrm{C}$ en el intercambiador de calor 1 , usando agua caliente, suministrada desde el tanque de agua caliente; este calentamiento se hace para ayudar a la propagación del cultivo bacteriológico de la siguiente etapa.

Descripción de Unidades. Unidad 1: Adecuación de leche [17]: En la unidad de adecuación de leche, se encuentra una serie de equipos para adecuar de la leche pasteurizada para producir yogurt. Lo primero es encontrar un tanque de almacenamiento, que contenga la leche pasteurizada, materia prima para producir de yogurt y, que proveerá la cantidad apropiada de leche para utilizar en la producción de un batch por medio de una bomba de suministro; en segundo lugar, la leche debe ser calentada por medio de un intercambiador de calor de placas que utiliza agua proveniente del tanque de agua caliente, mediante un sistema de bombeo, para elevar la temperatura de la leche a $42-43^{\circ} \mathrm{C}$. A continuación, se listan los equipos involucrados en esta unidad, y que son propios del proceso de fabricación de yogurt: tanque de almacenamiento (silo), intercambiador de calor 1 y tanque de almacenamiento de agua caliente.

Definición de módulos de equipo y módulos de control. Una vez identificados los equipos utilizados en la etapa de proceso, se definen los módulos de equipo y sus correspondientes módulos de control.

Módulo de equipo 1: equipo para transferir leche pasteurizada desde el tanque de almacenamiento.

1. Módulos de control 
2. Variables Controladas: Para garantizar que pase el flujo de leche deseado, se debe controlar el volumen de leche que sale del tanque de almacenamiento.

3. Variables manipuladas: La variable manipulada debe ser el flujo de leche que pasa por la válvula hacia el intercambiador de calor.

4. Disturbios: En la transferencia de leche, se puede presentar un disturbio: la variación del caudal de leche proveniente del tanque de almacenamiento hacia la zona de calentamiento; este disturbio se puede presentar debido a variaciones en el nivel del tanque de almacenamiento. Los cambios en el suministro de leche, afectarán el volumen de producción.

5. Clasificación disturbio: Este disturbio se considera no crítico, por que si se adiciona más o menos cantidad de leche, se puede modificar después los parámetros relevantes, de forma que se fabrique el tamaño del batch según la cantidad de leche que se transfirió.

6. Esquema de control: Conociendo el disturbio que afecta la etapa del proceso y las variables que se deben manipular y controlar se propone implementar un lazo de control feedback, en donde se mide el flujo de leche que sale del tanque de almacenamiento, de tal forma que se pueda determinar el volumen faltante de leche, según el tamaño del batch.

A partir del conocimiento de la instrumentación existente y del esquema de control por implementar, se determinan los módulos de control, como se lista a continuación:

- Módulo de Control 1: MC para suministro de leche pasteurizada.

- Módulo de Control 2: MC de transferencia de leche.

- Módulo de Control 3: Control de flujo de leche.

Módulo de equipo 2: Equipo para el calentamiento de leche pasteurizada.

1. Módulos de Control

2. Variables Controladas: En esta parte del proceso, se debe mantener la temperatura entre $42-43^{\circ} \mathrm{C}$. Por tal razón, se tiene como variable controlada la temperatura de la leche a la salida del intercambiador de calor 1 .

3. Variables Manipuladas: Para mantener dicha temperatura, se debe manipular el flujo de agua caliente que ingresa al intercambiador de calor 1.

4. Disturbios: En el calentamiento de leche, se puede presentar variaciones en: 1. la temperatura del agua caliente suministrada al intercambiador de calor 1, debido a que al tanque de almacenamiento retorna el agua que sale del intercambiador; esta variación afectaría significativamente el calentamiento de la leche. En el caso de que la leche se encuentre en una temperatura diferente del rango especificado, perjudicaría la propagación de las bacterias. 2. Flujo de agua caliente suministrada al intercambiador de calor 1, debido a variaciones en el nivel del tanque de agua caliente. 3. Variaciones en el flujo de leche que ingresa al intercambiador de calor 1, debido a variaciones de nivel en el tanque de almacenamiento. 4. Temperatura de la leche a la entrada del intercambiador de calor 1, por que en el tanque de almacenamiento no se tiene algún tipo de control. 5. Flujo de salida del intercambiador de calor 1, debido a cambios de flujo en la entrada. Sin 
embargo, los tanques de almacenamiento tanto de leche como de agua caliente, tienen salida de flujo constante.

5. Clasificación disturbio: El primer disturbio se considera crítico, ya que no se puede permitir la propagación de este disturbio, la temperatura de la leche no estaría en el valor adecuado para incubar las bacterias. El segundo disturbio se considera no existente, puesto que el tanque de agua caliente tiene salida de flujo constante. El tercer disturbio se considera no existente, el flujo de salida de los tanques de almacenamiento es constante. El cuarto disturbio también se considera no existente, pues la temperatura de la leche se asume ambiente. El quinto y último disturbio se clasifica también como no existente, por que no se tienen variaciones significativas a la salida del intercambiador de calor 1.

6. Esquema de control: Conociendo los disturbios que afectan la etapa del proceso, las variables que se deben manipular y controlar y que el disturbio es crítico se propone implementar un lazo de control feedforward, en donde se debe medir la temperatura del agua caliente suministrada al intercambiador, de tal forma que la acción de control permita el paso del flujo adecuado de agua caliente.

A partir del conocimiento de la instrumentación existente y del esquema de control por implementar, se determinan los módulos de control, como se lista a continuación:

- Módulo de Control 4: MC para transferencia de agua caliente.

- Módulo de Control 5: MC para retorno de agua caliente.

- Módulo de Control 6: Control de temperatura de leche.

Definición de operaciones y acciones de proceso. Una vez definidos los módulos de equipo y los módulos de control, se puede definir las Operaciones y Acciones dentro de la etapa de proceso Adecuación de Leche, de tal forma que las operaciones se encuentren asociadas a cada módulo de equipo; y las acciones se encuentren asociadas a los módulos de control, tal como se observa a continuación:

Operación 1.1: almacenamiento de leche, se encuentran las acciones:

- Traslado de leche a zona de almacenamiento.

Operación 1.2: suministro de leche hacia el proceso, cuyas acciones son

- Dosificación de leche según tamaño del batch.

- Traslado de leche a zona de calentamiento.

Operación 1.3: calentamiento de leche, compuesta por las acciones

- Aumento de temperatura de leche a $42-43^{\circ} \mathrm{C}$.

- Traslado de leche a tanques de fermentación.

Definición de modelos. En esta sección, se definen los modelos resultantes, a partir de la información obtenida, siguiendo el procedimiento: Modelo de proceso: Figura 7, Modelo físico: Figura 8. 


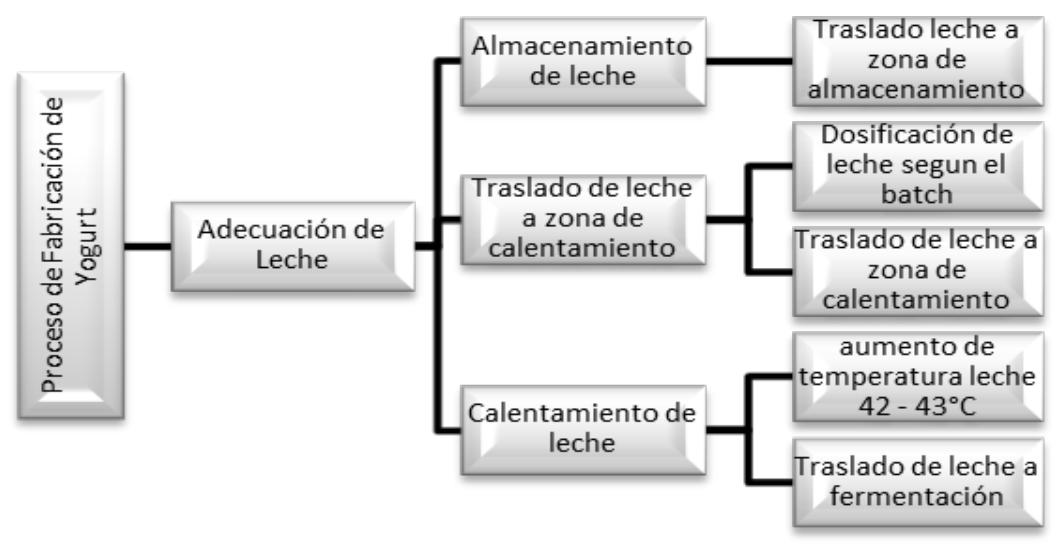

Figura 7. Modelo de proceso de la etapa de adecuación de leche del proceso de fabricación de yogurt

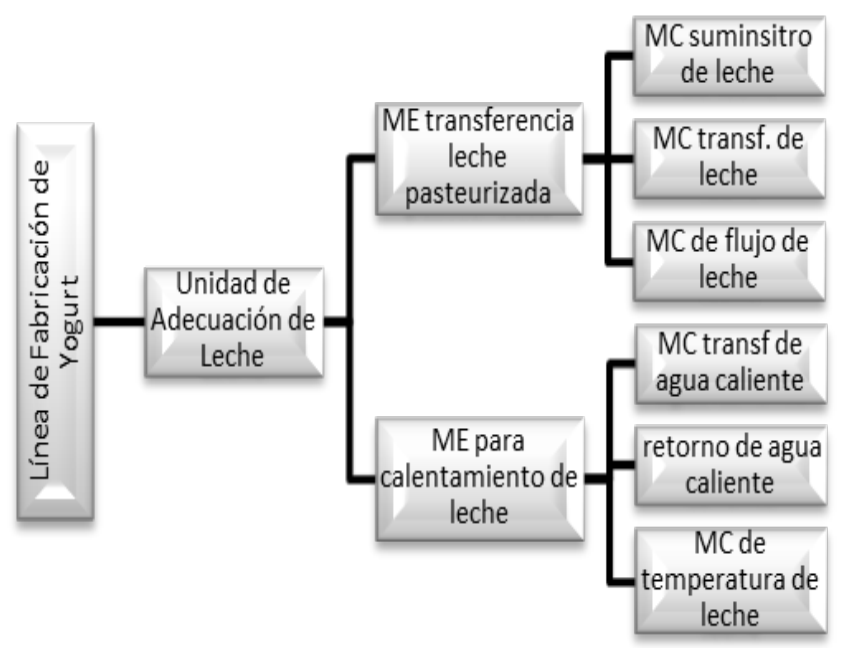

Figura 8. Modelo físico de la unidad de adecuación de leche en el proceso de fabricación de leche

Modelo de control procedimental. A partir de los modelos de proceso y físico, y las definiciones de la norma ISA S88, se procede a determinar cada componente del modelo de control procedimental y luego se realiza dicho modelo, Figura 9:

\section{Procedimiento: Fabricación de yogurt}

Procedimientos de unidad: Se definen los procedimientos de unidad, de acuerdo con las unidades definidas; en este caso, se definió la unidad Adecuación de leche, por lo tanto, se define el procedimiento de unidad: Adecuar Leche. 
Operaciones y Fases: Dentro del procedimiento de unidad adecuar leche, se puede definir la acción y sus respectivas fases así: Calentar Leche, la cual tiene dos fases: 1) Determinar leche por utilizar según tamaño de batch. 2) Aumentar temperatura de la leche a $42-43^{\circ} \mathrm{C}$.

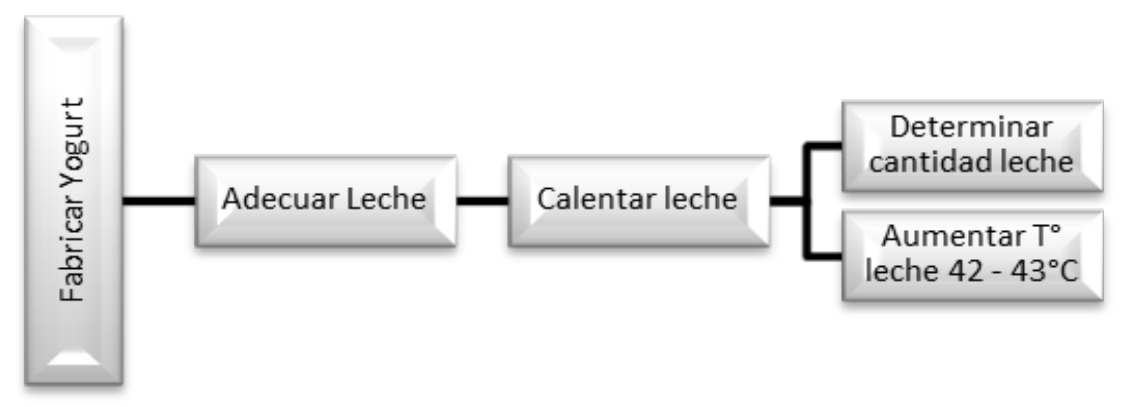

Figura 9. Modelo de control procedimental, para adecuar la leche del proceso de fabricación de yogurt

Diagrama P\&ID. Una vez definidos los tres modelos establecidos en ISA S88.01, con base en el procedimiento de adecuación de proceso, se puede definir el P\&ID, Figura 10.

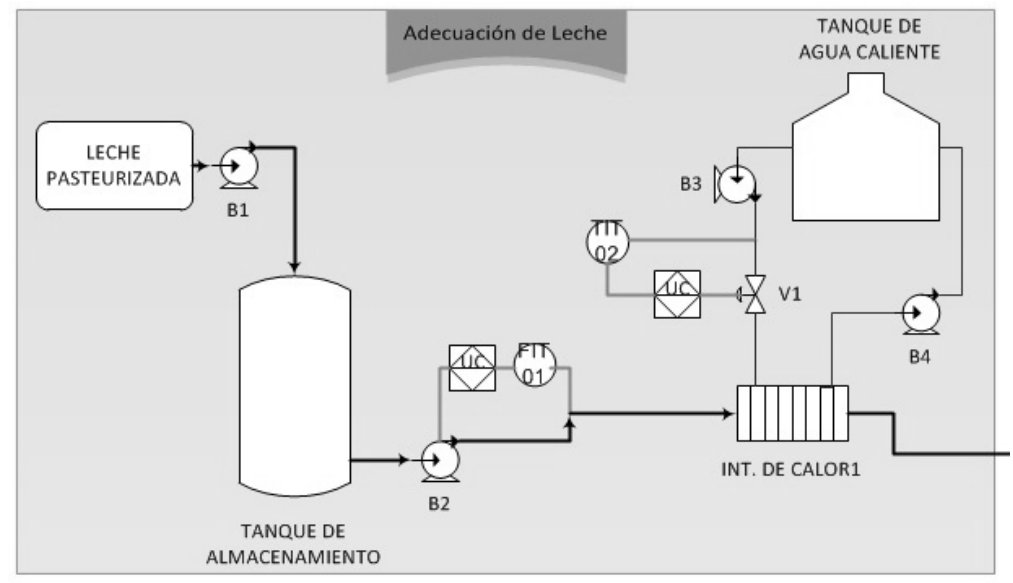

Figura 10. Diagrama P\&ID etapa adecuación de leche del proceso de fabricación de yogurt

Récipe Maestro. Para definir del récipe maestro, se definen los campos que hacen parte del mismo, a partir de las definiciones establecidas por ISA S88.01. Se debe especificar el encabezado, la fórmula, requerimientos de equipo y procedimiento.

\subsection{ESTRUCTURA DE DATOS CASO DE ESTUDIO}

A continuación se desarrolla el modelado de la estructura de datos para cada componente de récipe maestro del segmento de proceso adecuación de leche. 
Récipe Maestro para segmento de proceso adecuación de leche. En la Tabla 6, se define el modelo de datos del récipe maestro para el segmento de proceso adecuación de leche.

Tabla 6. Récipe Maestro segmento de proceso adecuación de leche

\begin{tabular}{|l|l|}
\hline \multicolumn{1}{|c|}{ Nombre } & \multicolumn{1}{c|}{ Récipe maestro } \\
\hline Descripción funcional & \multicolumn{1}{c|}{ Es un nivel de récipe dirigido a la célula de proceso. } \\
\hline \multicolumn{2}{|c|}{} \\
\hline ID Récipe & \multicolumn{1}{|c|}{ RMaestro_001 } \\
\hline Versión Récipe & 1.0 \\
\hline Fecha Versión & Mayo 10 de 2011 \\
\hline Fecha Aprobado & Mayo 15 de 2011 \\
\hline Fecha Efectiva & Junio 10 de 2011 \\
\hline Fecha de Expiración & \\
\hline ID Producto & LECHE_PAST_CAL \\
\hline Autor & $\begin{array}{l}\text { Diana Vergara } \\
\text { Werner Serna }\end{array}$ \\
\hline Aprobado por & $\begin{array}{l}\text { Diana Vergara } \\
\text { Werner Serna }\end{array}$ \\
\hline Descripción & \\
\hline Estado & Aprobado para producción. \\
\hline
\end{tabular}

Parámetros de récipe de segmento de proceso adecuación de leche. Parámetros de Entradas del Proceso: Se refieren a los materiales, materias primas, y aditivos que se utilizan durante el proceso de fabricación. En la Tabla 7, se define la entrada de proceso leche pasteurizada.

Tabla 7. Parámetro: Leche Pasteurizada

\begin{tabular}{|l|l|l|l|}
\hline \multicolumn{1}{|c|}{ Nombre } & \multicolumn{3}{c|}{ Leche pasteurizada } \\
\hline Descripción Funcional & Materia Prima Principal \\
\hline \multicolumn{3}{|c|}{ Atributos } \\
\hline ID Parámetro & MPLECHEPAST & Tipo de Parámetro & Constante \\
\hline Und. Ingeniería & Litros & Valor & 2.000 \\
\hline Escala & Escalable & Uso & Entrada de proceso \\
\hline Descripción & $\begin{array}{l}\text { El elemento más importante en la producción de yogurt, por que de ella } \\
\text { dependen muchas de las características finales del yogurt, como el contenido } \\
\text { graso y la consistencia. }\end{array}$ \\
\hline
\end{tabular}


Parámetros de Proceso: Se refieren a las especificaciones de proceso para tener en cuenta para garantizar que en el proceso se fabrique un producto con la calidad requerida. En la Tabla 8, se define el parámetro de proceso temperatura de leche pasteurizada.

Tabla 8. Parámetro: $T^{\circ}$ Leche Pasteurizada

\begin{tabular}{|l|l|l|l|}
\hline \multicolumn{1}{|c|}{ Nombre } & \multicolumn{3}{c|}{ T $^{\circ}$ leche pasteurizada } \\
\hline Descripción Funcional & \multicolumn{3}{|c|}{ Temperatura de preparación para la propagación de bacterias } \\
\hline \multicolumn{4}{|c|}{ Atributos } \\
\hline ID Parámetro & PARTLECHEP & Tipo Parámetro & Referencia \\
\hline Und. ingeniería & ${ }^{\circ} \mathrm{C}$ & Valor & $42-43^{\circ} \mathrm{C}$ \\
\hline Escala & No escalable & Uso & Parámetro de Proceso \\
\hline Descripción & $\begin{array}{l}\text { Temperatura de preparación que ayuda a la propagación del cultivo de } \\
\text { bacterias utilizadas en la fermentación de la leche, ya que al tener la } \\
\text { temperatura adecuada, los tiempos de fermentación se reducen. }\end{array}$ \\
\hline
\end{tabular}

Parámetros Salidas de Proceso: Se refieren a los productos finales e intermedios que resultan durante y al final del proceso de fabricación. Para el caso del segmento de proceso, se tiene como salida de proceso: leche pasteurizada caliente, que se encuentra definido en la Tabla 9.

Tabla 9. Parámetro: Leche Pasteurizada a $42^{\circ} \mathrm{C}$ a $43^{\circ} \mathrm{C}$

\begin{tabular}{|l|l|l|l|}
\hline \multicolumn{1}{|c|}{ Nombre } & \multicolumn{3}{|c|}{ Leche pasteurizada caliente } \\
\hline Descripción Funcional & Producto final \\
\hline \multicolumn{3}{|c|}{ Atributos } \\
\hline ID Parámetro & PF_LECHPAST & Tipo de Parámetro & Constante \\
\hline Und. de Ingeniería & ${ }^{\circ} \mathrm{C}$ & Valor & 42 - 43 \\
\hline Escala & No Escalable & Uso & Salida de Proceso \\
\hline Descripción & $\begin{array}{l}\text { Producto lácteo, calentado con el uso de un intercambiador de calor, con el fin } \\
\text { de propiciar las condiciones adecuadas para la propagación de las bacterias. }\end{array}$ \\
\hline
\end{tabular}

\subsection{LINEAMIENTOS DE LENGUAJE CASO DE ESTUDIO}

En esta sección se definen los componentes del diagrama PFC, aplicando el procedimiento establecido en la sección 0 Lineamientos de lenguaje

Fases de procedimiento de récipe. En la Figura 10, se puede observar las fases de procedimiento de récipe para el segmento de proceso adecuación de leche. Se enumeran de 1 a 2 para tener un orden en el momento de realizar el diagrama para las operaciones. 


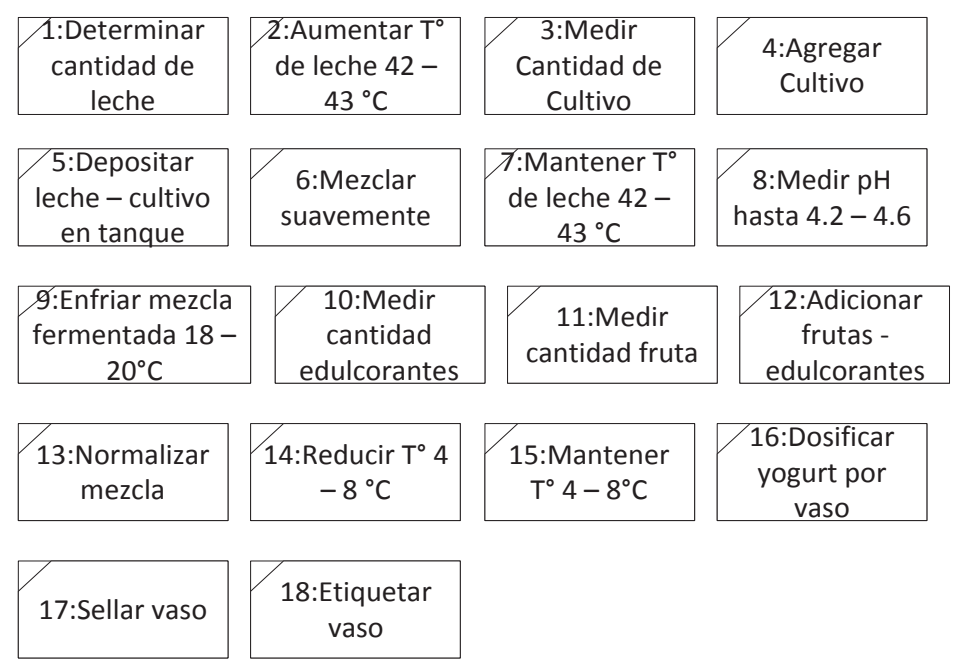

Figura 10. Representación de diagrama PFC de las fases de procedimiento de récipe maestro para el proceso de producción de yogurt

Operaciones de procedimiento de Récipe. Después de tener la representación PFC de las fases, se seleccionan las fases de cada operación, según el procedimiento de récipe maestro, de forma que se encapsulen las fases en su respectiva operación. En la Figura 11. se encuentra definida la operación Calentar leche que contiene las fases 1 y 2.

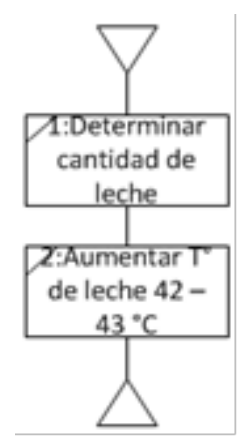

Figura 11. Diagrama PFC de la operación calentar leche

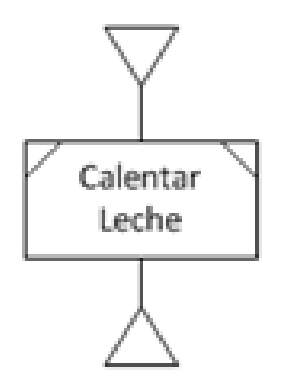

Figura 12. Diagrama PFC del procedimiento de unidad

Con los diagramas PFC de las operaciones, se procede a realizar el diagrama PFC del procedimiento de unidad. En la Figura Figura 12 Se puede observar dicho diagrama:

Al tener los diagramas PFC de todos los procedimientos de unidad, se procede a realizar el diagrama PFC del procedimiento de récipe; en la Figura 13, se puede observar dicho diagrama. Una vez realizados todos los diagramas PFC desde las fases hasta el procedimiento de récipe, se concluye el procedimiento de modelado ISA S88. Se puede editar en herramientas software 
especializadas, la ejecución de órdenes de producción basadas en récipes. Con el desarrollo de los procedimientos propuestos, se determinan los modelos y la información de una manera adecuada, y se prepara así para la edición en herramientas software.

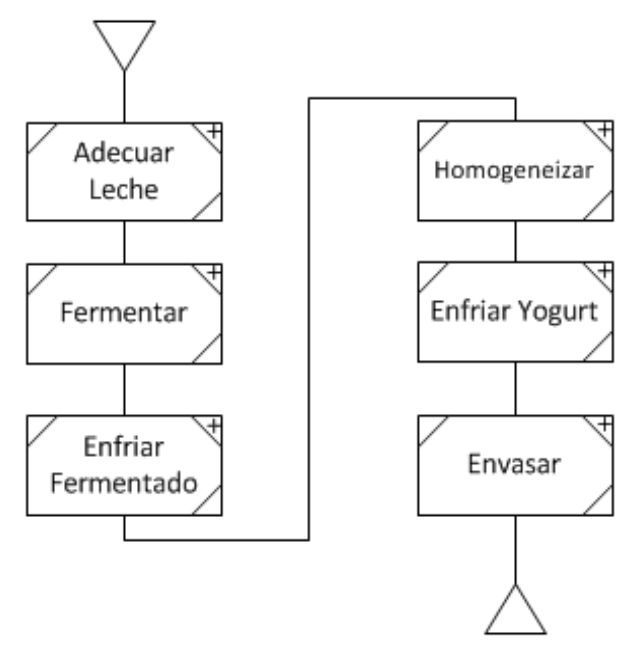

Figura 13. Diagrama PFC de Procedimiento de Récipe Maestro

\section{CONCLUSIONES}

En la automatización de procesos industriales, se considera importante aplicar normas internacionales que permitan normalizar los procesos. Normas como las establecidas por ISA, ayudan al logro de este propósito, pero la ausencia de un procedimiento que permita aplicar dichas normas, es un inconveniente que se presenta en la empresas integradoras colombianas. En el presente trabajo, se ha propuesto una serie de procedimientos que ayudan al seguimiento y aplicación de los estándares internacionales ISA S88 parte 1 y 2.

Una de las normas utilizadas en la automatización de los procesos industriales es la ISA S88 para procesos tipo batch, que establece en su parte 1, una serie de modelos de proceso, físico y de control procedimental, además de cuatro récipes que ayudan a la estandarización de los procesos. El primer procedimiento desarrollado en este trabajo, está enfocado en la aplicación de ISA S88.01, y consta de 11 pasos a partir de los cuales se obtienen los modelos y récipes establecidos por dicha norma.

La ISA S88 propone una segunda parte que permite estandarizar la información del producto, información que se debe tener en cuenta en el uso de herramientas software industriales que permite ejecutas órdenes de producción a partir de récipes, basándose en los modelos definidos en la parte 1. Esto se realiza mediante las estructuras de datos y los lineamientos de lenguaje que se encuentran definidos en dicha parte de la norma. El segundo procedimiento 
desarrollado sugiere siete pasos para tener en cuenta en la definición de los modelos establecidos en ISA S88.02, los cuales permiten especificar la información del producto con los requerimientos particulares del nivel de planta. Anteriormente, se definían, utilizando la norma ISA S95 cuyo enfoque es el nivel de gestión, por lo cual se consideraba información que no era necesaria en la ejecución de un batch.

La serie de pasos procedimentales que se proponen en el presente trabajo para aplicar las partes 1 y 2 de la norma ISA S88, con la cual se modelan procesos industriales tipo batch, permite definir modelos de tal forma que no se presenten inconsistencias entre ellos, además de organizar y preparar la información necesaria para el uso de herramientas software que permiten ejecutar órdenes de producción basadas en el récipe del producto por fabricar.

La definición de un procedimiento sistemático para el modelado de la norma ISA S88, permite realizar una forma generalizada los modelos de dicha norma, es decir, se obtienen modelos que serán interpretados de la misma manera por aquellas personas que hagan uso del procedimiento.

\section{AGRADECIMIENTOS}

Se agradece a la Universidad del Cauca y al grupo de I+D en Automática Industrial por la colaboración y el apoyo en el desarrollo del proyecto, por facilitar los medios y recursos que permitieron darle culminación al presente trabajo.

\section{REFERENCIAS BIBLIOGRÁFICAS}

[1] Instrument Society of America. ANSI/ISA-88.01-1995, Batch Control, Part 1: Models and Terminology. Norma Técnica. Instrument Society of America, Triangle Park, North Carolina, 1995.

[2] Instrument Society of America. ANSI/ISA-95, Enterprise-Control System Integration. Norma Técnica, Instrument Society of America, Triangle Park, North Carolina, 2000.

[3] REDES ELÉCTRICAS S.A. Bogotá: DESARROLLO Y ADMINISTRACIÓN POR REDES ELÉCTRICAS S.A, 2010 citado 22 mayo de 2010. Actualización tecnológica del sistema de manejo de materiales a granel por lotes Reimelt - Bimbo de Colombia S.A. En: http://mww.redeselectricas.com/paginas/proyectos/bimbo.html

[4] Szklanny, S.V. y Pasmanik, D. Aplicando la Norma ISA S88: el futuro inmediato del control secuencial (Batch - por lotes), Beneficios y Necesidades.

[5] CHACÓN, Édgar et al. Aplicación del Estándar ISA88 en el Modelado del Proceso de Producción de Azúcar en un Central Azucarero, LACCEI 2009. 
[6] RÚlZ, Julio. Modelo de un tacho batch al vacío para agotamiento de mieles provenientes de la Caña de Azúcar. Tesis Doctoral. Atlantic International University. Miami, 2008.

[7] The WBF series. Applyng isa-88 in discrete and continuous manufacturing. 2010. Vol 1: 39-61. En: http://books.google.com.co/books? id $=$ klLqTxGScpEC\&printsec $=$ frontcover\&dq $=i s a+88 \& h l=e s \& s a=X \& e i=Y S-$ sdfsdf.

[8] Peña, Johanna. Modelización isa-88 y diagnóstico de fallos de Sistemas Control de Riego. Tesis maestría. Universidad politécnica de Cataluña, 2010.

[9] Ingeniero en Omnicon. Entrevista personal realizada en marzo de 2010. Omnicon S.A. Solution Provider Rockwell Automation en Colombia.

[10] Instrument Society of America. ANSI/ISA-95 Enterprise Control System Integration. Norma Técnica, Instrument Society of America, Triangle Park, North Carolina, 2005.

[11] Soluciones Wonderware Online. 2006. Invensys Wonderware Inbatch. Soluciones Wonderware - InBatch. [Citado junio 21 de 2011]. En: http://mww.logiteksa.com/contents/sw/productos/inbatch.html

[12] ARC Advisory Group. 2005. Siemens.nl. Siemens Process Industry Strategies. [Citado junio 21 de 2011]. En: http://mww.industry.siemens.nl/automation/nl/nl/industrieleautomatisering/PCS7/Documents/Whitepaper_ARC_ProcessControl_PCS7.pdf.

[13] Serna, Werner Yamid y Vergara, Diana. Solución de integración hacia un nivel de gestión para un proceso tipo batch, basada en ISA S88. Tesis Pregrado. Universidad del Cauca. Popayán, 2011.

[14] Velasco, Juan Martín. Notas de Clase. Cad / Cam / Cim. Universidad del Cauca. Popayán, 2008.

[15] Flórez, Juan Fernando. Notas de Clase. Instrumentación Industrial. Universidad del Cauca, Popayán, 2007.

[16] Instrument Society of America. ANSI/ISA-88.02-1995, Batch Control, Part 2: Data Structures and Guidelines for Languages. Norma Técnica. Instrument Society of America, Triangle Park, North Carolina, 2001.

[17] Tetra Pak Processing Systems AB. Productos lácteos acidificados. En: Manual de Industrias Lácteas. Traducido por: López Gómez, Antonio 3ed. USA. Madrid Vicente, Antonio: Tetra Pak, 2003, pp.241 y 254. 
